\title{
Risk Management under time varying volatility and Pareto Stable distributions
}

Sharif Mozumder ${ }^{\mathrm{a}}$, M. Humayun Kabir ${ }^{\mathrm{b}}$, Michael Dempsey ${ }^{\mathrm{c}}$, Taufiq Choudhry ${ }^{\mathrm{d}}$,

${ }^{a}$ University of Dhaka - Department of Mathematics

${ }^{d}$ School of Economics \& Finance, Massey University, New Zealand

${ }^{\mathrm{c}}$ School of Economics, Finance and Marketing, RMIT University, Australia

${ }^{d}$ School of Business, University of Southampton, UK 


\title{
Risk Management under time varying volatility and Pareto Stable distributions
}

\begin{abstract}
Risk measures based on Gaussian return distributions are simple but inaccurate while such measures based on alternative methodologies are known to be more precise but complex. In this context, practitioners seem biased towards simplicity and tend to choose the inaccurate Gaussian measures, leading to unsuspected losses in the event of a negative episode. This paper proposes a change to the conventional $\mathrm{VaR}$ approach that promises a markedly improved performance while maintaining simplicity.
\end{abstract}

JEL-classification:

Keywords: GARCH models, Stable Processes, Risk Measures, Stable Paretian Distribution 


\section{Introduction}

Practitioners seek reliable measures of risk that are at the same time not overly demanding to understand and implement. The common measures used in risk management such as Value-atRisk (VaR) are based on the assumption that return distributions follow independent and identically distributed (iid) Gaussian processes. However, the financial data typically exhibit serial dependence, volatility clustering and leptokurtosis characteristics. The issues of timevarying mean and time-varying variance are partially addressed through ARCH (Engle, 1982) and GARCH (Bollerslev, 1986) models. The leptokurtic character of financial data, however, is ignored in most cases. In crisis periods, the outcomes are likely to be characterized by leptokurtic return distributions. Thus, an effective risk management require tail-based risk measures based on a methodology that recognizes the leptokurtic character of financial returns. In this context, GARCH models with non-normal innovations offer a promising tool. While Bollerslev (1987) suggests the Student's $t$ distribution, Nelson (1991) and Granger and Ding (1995) propose GED, and the Laplace distribution respectively.

In this paper, we propose the use of GARCH family models with Stable Paretian innovations to account for the non-stationary nature of the data. The Stable Paretian distribution, first proposed by Mandelbrot (1963), can provide accurate prediction of volatility ${ }^{1}$. Using the return series leading equity indices, we estimate standard GARCH, EGARCH and GJR-GARCH models to capture the dynamics of returns volatility and derive time-varying volatilities. These volatilities are used as proxies to the scale parameter of the returns distribution to estimate different measures of risk.

\footnotetext{
1 This has been demonstrated by Mittnik et al. (1988), Liu and Brorsen (1995), Panorska et al. (1995), and Mittnik and Paolella (2003).
} 
We consider VaR, Expected Shortfall (ES) and Spectral Risk (SRM) measures and compare their performance with the same measures derived assuming a constant volatility approach. Our results show that the risk estimates based on the proposed GARCH family models with Stable Paretian innovations perform much better than their constant volatility counterpart. Our approach provides for an improved risk approximation without undue computational burden.

This paper is organized as follows. In section 2, we discuss data and methodology related to different GARCH dynamics under SP innovations. Section 3 presents the preliminary statistics of the data and estimation results. Section 4 concludes the paper.

\section{Data and methodology}

The data consists of daily closing prices of worldwide leading equity market indexes such as the S\&P500, the FTSE100, and the DAX from $4^{\text {th }}$ August, 2008 to 31 st December 2017. Thus our sample covers the period of global financial crisis period for which the risk measures are more relevant. Returns are calculated by taking log-differences of the closing prices and expressed in percentage rates.

We use three benchmark measures of risk based on VaR. The first one is the conventional VaR founded on Gaussian assumptions such that $r \sim N\left(0, \sigma^{2}\right)$ :

$$
\operatorname{Pr}[P(t+\tau)-P(t)<-V a R]=1-\alpha
$$

where, $\alpha$ is the confidence level and $P(t+\tau)-P(t)$ is the log return of the portfolio over the time horizon $\tau$. In case of a single asset, VaR would be then calculated as:

$$
1-\alpha=\int_{-\infty}^{z_{1-\alpha}} \phi(z) d z=N\left(z_{1-\alpha}\right)
$$


where, $\phi(z)$ is the normal density function, $N(z)$ is the cumulative normal distribution function and $\alpha$ is the relevant confidence level. Assuming mean, $\mu=0$, VaR can be defined as:

$$
V a R=-V_{0} z_{1-\alpha} \sigma
$$

where $V_{0}$ is the initial portfolio value.

In addition to the conventional VaR, we use two additional risk measures which meet desirable characteristics such as coherence and sub-additivity. We consider the conventional Expected Shortfall (ES) measure, which considers the whole tail of losses, given as follows:

$$
E S(\alpha)=\frac{1}{1-\alpha} \int_{\alpha}^{1} \operatorname{VaR}(v) d v,
$$

where $\alpha$ is the coverage level.

Next, we consider the spectral risk measure (SRM) proposed by Acerbi (2002). The main characteristic of this measure is that it does not rely on any particular confidence level. Given the degree of investor risk aversion, SRM considers the whole spectrum of losses. Based on the exponential risk aversion function, the SRM can expressed as:

$$
M_{\phi}(R)=\int_{0}^{1} \frac{R e^{-R(1-\alpha)}}{1-e^{-R}} \operatorname{VaR}(v) d v \text {, }
$$

where $\phi$ denotes the risk aversion function and the other variables are as previously defined. ${ }^{2}$

As reflected in equations (4) and (5), these risk measures are based on VaR, so that they are equally dependent on the constant volatility. Since the assumption of constant scale is not realistic, the estimates of this parameter based on GARCH family models with SP innovations should result in more accurate measures. We use traditional GARCH as well as E-GARCH and

\footnotetext{
${ }^{2}$ Additional choices for risk aversion functions are discussed in Dowd and Cotter (2006).
} 
GJR-GARCH models as alternatives for time-varying volatility. Formally, a GARCH with SP innovations (GARCH-SP herein) can be written as follows:

$$
\begin{gathered}
r_{t}=\delta+\varepsilon_{t}=\delta+\eta_{t} \sqrt{h_{t}}, \\
h_{t}=\alpha_{0}+\alpha_{1} \varepsilon_{t-1}^{2}+\beta h_{t-1},
\end{gathered}
$$

where, $\alpha_{0}>0, \alpha_{1}>0, \beta_{1} \geq 0$ to ensure that conditional variance always remain positive. In the model, $\sqrt{h_{t}}$ is the scale parameter of the process at time $t$, and the innovation, $\varepsilon_{t}$ in this dynamics is such that $\varepsilon_{t}=\eta_{t} \sqrt{h_{t}}$, with $\eta_{t}$ iid as realizations of a stable Paretian distributed random variable given $\alpha>1 .^{3}$

Under the non-Gaussian assumptions justifying the use of GARCH model, the VaR is calculated following equation (2) but considering a non-constant volatility. In this framework, VaR is now estimated as:

$$
\operatorname{VaR}=-V_{0} z_{1-\alpha} \sigma_{\text {proxy }}
$$

where $\sigma_{\text {proxy }}$ is the volatility estimate provided by the GARCH-SP model. Given the fact that we only consider cases where there is a solution of the VaR, the ES and SRM measures can simply be derived following equations (5) and (6).

To that end, we first estimate the empirical measures based on the whole sample and, then follow a parametric bootstrap approach with 5,000 resamples to simulate VaR values based on time-varying scale parameter. For each resample we draw uniform random numbers of same size that, sorted in ascending order, which can be considered as a set of resampled cumulative probabilities. We then find the relevant quantile corresponding to the particular

\footnotetext{
${ }^{3}$ Notice that $\eta_{t}$ iid $\sim S_{\alpha, \beta} . S_{\alpha, \beta}$ represents the standard asymmetric stable Paretian distribution with stable index $\alpha \in(0,2]$, skewness parameter $\beta \in[-1,1]$, zero location parameter and unit scale parameter. We use the characteristic function corresponding to this model is given by Samorodnitsky and Taqqu (1994) and Rachev and Mittnik (2000).
} 
coverage level, and calculate VaR and ES following equations (4) and (5). As the SRM does not depend on the coverage level, it is necessary to randomize the whole spectrum to obtain bootstrapped estimates. We approximate the integral in equation (7) by slicing the spectrum. ${ }^{4}$ For these estimations we restrict ourselves to bootstrapping with 100 resamples. Following these procedures, we are able to estimate VaR, ES and SRM, the standard errors (SE) and the confidence intervals (CI) for each market index and different coverage levels.

\section{Empirical Findings and Discussions}

Table 1 summarizes basic statistical properties of the data. The mean returns are all positive but close to zero and their distributions appear to be asymmetric as reflected by negative skewness. These features are further confirmed by the Jarque-Bera (1987) test. Moreover, the Ljung-Box statistic of squared returns is highly significant at any level for the three stock indexes suggesting the presence of strong nonlinear dependences in the data.

\section{[Table 1]}

Both the nonlinear dependence and the heavy-tailed nature of these unconditional returns distributions suggest the possibility that $\mathrm{ARCH}$ effects are predominant in our data. The Lagrange Multiplier (LM) of test Engle (1982) suggests the presence of ARCH effects in the variance of the processes (Hsieh 1989). In summary, our descriptive statistics confirm the recognized stylized facts of the returns distribution and highlight the necessity of incorporating GARCH model families in order to capture the effects of conditional heteroskedasticity in the distributions. We estimate the parameters of GARCH, EGARCH and GJR-GARCH models for each of the sample market indexes.

The results of risk measures are presented in Tables 2 to 5 . The tables show the estimates of VaR, ES at $1 \%, 0.5 \%$ and $0.1 \%$ coverage levels and those for SRM based on 50,

\footnotetext{
${ }^{4} \mathrm{We}$ follow this approach because randomisation of the integral often fails to converge under numerical schemes such as one used by matlab function 'quadl'.
} 
100 and 200 resamples for the randomization of the spectrum. Each table corresponds to one of the three market indexes in two panels: Panel A corresponds to the risk measures estimates based on a long position, and Panel B for a short position. The standard errors are reported in the parenthesis with confidence interval between brackets.

We find that the risk measures based on non-Gaussian estimation outperform their Gaussian peers. Only in 2 of the 216 cases are the constant volatility risk measures closer to the empirical values than are its peers based on GARCH models with SP innovations (Table 4 Panel B). In addition, VaR under constant volatility is $28 \%$ lower than the empirical outcomes, which is only slightly better than the $29 \%$ for the GJR model and the $30 \%$ for the GARCH and EGARCH models. In the case of the S\&P500 index (Table 2), the average gain in using the proposed non-Gaussian approach is $8 \%$, which is significant in economic terms. Our approach seems especially relevant in the case of the FTSE 100 index (Table 3), where the average gains of our non-Gaussian risk measure estimates are of $13 \%$, highlighting the fact that there are layers of information that are not exploited by the conventional risk measures. We find results similar to S\&P500 index for the DAX index in Table 4.

\section{Conclusion}

Despite the fact that more precise models and methodologies have been developed in recent years in estimating risk measures, this task is still commonly tackled relying on estimates based on the Normal distribution. In this paper, we have proposed a subtle change to the conventional VaR approach based GARCH family models that are familiar to practitioners. Our approach outperforms the conventional approach in each of the markets of our study. Our risk estimates are as much as $19 \%$ closer to the true ones than those offered by conventional risk measures. Given its simplicity, we believe our approach has significant application for practitioners. 


\section{References:}

Acerbi, C., 2002. Spectral measures of risk: A coherent representation of subjective risk aversion. Journal of Banking and Finance 26, 1505-1518.

Cotter, J., Dowd, K. 2006. Extreme spectral risk measures: An application to futures clearinghouse margin requirements. Journal of Banking and Finance.30, 3469-3485.

Dowd, K., Cotter, J., Sorwar, C. 2008. Spectral Risk Measures: Properties and Limitations. Journal of Financial Services and Research. 34, 61-75.

Bollerslev, T. 1986. Generalized autoregressive conditional heteroskedasticity. Journal of Econometrics, 31:307-327

Bollerslev, T. 1987. A conditional heteroskedastic time series model for speculative prices and rates of return. Review of Economics and Statistics, 69:542-547

Engle, R.F. 1982. Autoregressive conditional heteroskedasticity with estimates of the variance of United Kingdom inflation. Econometrica 50(4):987-1006

Hsieh, D.A. 1989. The statistical properties of daily foreign exchange rates: 1974-1983. Journal of International Economics, 24:129- 145

Jarque, C. M., Bera, A.K. 1987. A test for normality of observations and regression residuals. International Statistical Review 55(2):163-172

Liu, S.M., Brorsen, B.W. 1995. Maximum likelihood estimation of a GARCH-stable model. Journal of Applied Econometrics, 10:273-285

Mandelbrot, B. 1963. The variation of certain speculative prices. Journal of Business, 36:394-419

Mittnik, S., Paolella, M.S., Rachev, S.T. 1998. Unconditional and conditional distributional models for the Nikkei Index, Asia Pacific Financial Markets, 5:99-128

Mittnik, S., Paolella, M.S. 2003. Prediction of financial downside risk with heavy-tailed conditional distributions. In: Handbook of heavy tailed distributions in finance, Vol 1, Chap 9, Elsevier/North-Holland, New York.

Nelson, D.B. 1991. Conditional heteroskedasticity in asset returns: a new approach. Econometrica, 59(2):347-370

Panorska, A., Mittnik, S., Rachev, S.T. 1995. Stable GARCH models for financial time series. Applied Mathematics Letter, 8:33-37

Rachev, S., Mittnik, S. 2000. Stable Paretian models in finance. Wiley, New York Samorodnitsky, G., Taqqu, M.S. 1994. Stable non-Gaussian random processes, stochastic models with infinite variance. Chapman and Hall London 
Table 1: Descriptive statistics

\begin{tabular}{|c|c|c|c|c|c|c|c|c|c|c|c|c|}
\hline Index & & No. of obs & Max & Min & Mean & Std dev & Skewness & Kurtosis & LM & $\mathrm{JB}$ & LB & LB2 \\
\hline \multirow[t]{2}{*}{ S\&P500 } & Long & 2369 & 9.47 & -10.96 & -0.032 & 1.57 & 0.32 & 11.52 & 53.03 & 4105.91 & 84.72 & 2046.39 \\
\hline & Short & 2369 & 10.96 & -9.47 & 0.032 & & -0.32 & & & & & \\
\hline \multirow[t]{2}{*}{ FTSE100 } & Long & 2376 & 9.26 & -9.38 & -0.015 & 1.42 & 0.10 & 10.64 & 70.74 & 3296.99 & 63.30 & 1611.08 \\
\hline & Short & 2376 & 9.38 & -9.26 & 0.015 & & -0.10 & & & & & \\
\hline \multirow[t]{2}{*}{ DAX } & Long & 2386 & 7.34 & -10.72 & -0.029 & 1.64 & -0.15 & 8.70 & 32.96 & 1850.96 & 42.34 & 1097.51 \\
\hline & Short & 2386 & 10.72 & -7.34 & 0.029 & & 0.15 & & & & & \\
\hline
\end{tabular}

Note: Data consists of returns from $4^{\text {th }}$ August 2008 to 31st December 2017 for S\&P500, FTSE100 and DAX indexes. No. of Obs. stands for number of observations available in the sample period. Std. dev. corresponds to the standard deviation of the return distributions. LM is the Lagrange Multiplier statistic, JB stands for the Jarque-Bera statistic, and LB stands for the Ljung-Box statistic.

Table 2: Risk estimate for S\&P500

\begin{tabular}{|c|c|c|c|c|c|c|c|c|c|}
\hline Model & $\operatorname{VaR}(0.99)$ & $\mathrm{ES}(0.99)$ & $\operatorname{SRM}(50)$ & $\operatorname{VaR}(0.995)$ & $\mathrm{ES}(0.995)$ & $\operatorname{SRM}(100)$ & $\operatorname{VaR}(0.999)$ & $\mathrm{ES}(0.999)$ & $\operatorname{SRM}(200)$ \\
\hline \multicolumn{10}{|c|}{ Panel A: Long position } \\
\hline Empirical & 4.2577 & 6.2854 & 4.6513 & 5.9228 & 7.6154 & 5.9855 & 10.3532 & 10.9572 & 7.6447 \\
\hline \multirow{2}{*}{$\sigma_{\text {constant }}$} & $3.673(0.159)$ & $4.203(0.145)$ & $3.547(0.206)$ & $4.064(0.199)$ & $4.558(0.178)$ & $3.958(0.323)$ & $4.870(0.366)$ & $5.295(0.333)$ & $4.339(0.486)$ \\
\hline & {$\left[\begin{array}{ll}0.932 & 1.074\end{array}\right]$} & {$\left[\begin{array}{ll}0.945 & 1.059\end{array}\right]$} & {$\left[\begin{array}{ll}0.901 & 1.099\end{array}\right]$} & {$\left[\begin{array}{ll}0.921 & 1.084\end{array}\right]$} & {$\left[\begin{array}{ll}0.938 & 1.068\end{array}\right]$} & {$\left[\begin{array}{ll}0.868 & 1.137\end{array}\right]$} & {$\left[\begin{array}{ll}0.886 & 1.136\end{array}\right]$} & {$[0.904$ 1.112] } & {$\left[\begin{array}{ll}0.815 & 1.191]\end{array}\right]$} \\
\hline \multirow[t]{2}{*}{$\sigma_{G A R C H-S P}$} & $3.9004(0.199)$ & $4.570(0.180)$ & $3.742(0.296)$ & $4.394(0.249)$ & $5.018(0.231)$ & $4.261(0.461)$ & $5.412(0.467)$ & $5.950(0.426)$ & $4.741(0.691)$ \\
\hline & {$\left[\begin{array}{ll}0.919 & 1.089\end{array}\right]$} & {$\left[\begin{array}{ll}0.937 & 1.067\end{array}\right]$} & {$\left[\begin{array}{ll}0.907 & 1.096\end{array}\right]$} & {$\left[\begin{array}{ll}0.911 & 1.020\end{array}\right]$} & {$\left[\begin{array}{ll}0.921 & 1.080\end{array}\right]$} & {$\left[\begin{array}{ll}0.868 & 1.138\end{array}\right]$} & {$\left[\begin{array}{ll}0.871 & 1.154\end{array}\right]$} & {$\left[\begin{array}{ll}0.891 & 1.130\end{array}\right]$} & {$\left[\begin{array}{ll}0.818 & 1.191]\end{array}\right]$} \\
\hline \multirow[t]{2}{*}{$\sigma_{E G A R C H-S P}$} & $3.593(0.327)$ & $4.671(0.290)$ & $3.337(0.618)$ & $4.387(0.405)$ & $5.391(0.370)$ & $4.173(0.943)$ & $6.025(0.759)$ & $6.894(0.676)$ & $4.946(1.395)$ \\
\hline & {$[0.858 \quad 1.155]$} & {$\left[\begin{array}{ll}0.901 & 1.105\end{array}\right]$} & {$\left[\begin{array}{ll}0.908 & 1.094\end{array}\right]$} & {$\left[\begin{array}{ll}0.851 & 1.161\end{array}\right]$} & {$\left[\begin{array}{ll}0.888 & 1.118\end{array}\right]$} & {$\left[\begin{array}{ll}0.870 & 1.133\end{array}\right]$} & {$\left[\begin{array}{ll}0.809 & 1.227\end{array}\right]$} & {$\left[\begin{array}{ll}0.850 & 1.181\end{array}\right]$} & {$\left[\begin{array}{ll}0.821 & 1.188\end{array}\right]$} \\
\hline \multirow[t]{2}{*}{$\sigma_{G J R-S P}$} & $3.649(0.315)$ & $4.703(0.284)$ & $3.398(0.598)$ & $4.426(0.395)$ & $5.409(0.361)$ & $4.217(0.901)$ & $6.029(0.750)$ & $6.879(0.674)$ & $4.973(1.370)$ \\
\hline & {$[0.8601 .144]$} & {$\left[\begin{array}{ll}0.904 & 1.104\end{array}\right]$} & {$\left[\begin{array}{ll}0.908 & 1.094\end{array}\right]$} & {$\left[\begin{array}{ll}0.856 & 1.157\end{array}\right]$} & {$[0.8941 .116]$} & {$[0.8731 .132]$} & {$\left[\begin{array}{ll}0.811 & 1.230\end{array}\right]$} & {$\left[\begin{array}{ll}0.851 & 1.178\end{array}\right]$} & {$\left[\begin{array}{ll}0.824 & 1.192\end{array}\right]$} \\
\hline \multicolumn{10}{|c|}{ Panel B: Short position } \\
\hline Empirical & 5.038 & 6.896 & 5.093 & 6.307 & 8.017 & 6.442 & 9.371 & 9.470 & 7.956 \\
\hline \multirow[t]{2}{*}{$\sigma_{\text {constant }}$} & $3.620(0.162)$ & $4.150(0.140)$ & $3.494(0.205)$ & $4.011(0.197)$ & $4.505(0.181)$ & $3.906(0.319)$ & $4.817(0.374)$ & $5.243(0.326)$ & $4.286(0.497)$ \\
\hline & {$\left[\begin{array}{ll}0.928 & 1.075\end{array}\right]$} & {$\left[\begin{array}{ll}0.946 & 1.058\end{array}\right]$} & {$\left[\begin{array}{ll}0.904 & 1.097\end{array}\right]$} & {$\left[\begin{array}{ll}0.921 & 1.084\end{array}\right]$} & {$\left[\begin{array}{ll}0.935 & 1.069\end{array}\right]$} & {$\left[\begin{array}{ll}0.872 & 1.137\end{array}\right]$} & {$\left[\begin{array}{ll}0.884 & 1.141\end{array}\right]$} & {$\left[\begin{array}{ll}0.905 & 1.113\end{array}\right]$} & {$\left[\begin{array}{ll}0.817 & 1.192\end{array}\right]$} \\
\hline \multirow[t]{2}{*}{$\sigma_{G A R C H-S P}$} & $3.507(0.145)$ & $3.991(0.129)$ & $3.392(0.179)$ & $3.863(0.179)$ & $4.314(0.163)$ & $3.767(0.278)$ & $4.599(0.338)$ & $4.987(0.303)$ & $4.114(0.436)$ \\
\hline & {$\left[\begin{array}{ll}0.934 & 1.071]\end{array}\right.$} & {$\left[\begin{array}{ll}0.949 & 1.054\end{array}\right]$} & {$\left[\begin{array}{ll}0.905 & 1.099\end{array}\right]$} & {$\left[\begin{array}{ll}0.924 & 1.081\end{array}\right]$} & {$\left[\begin{array}{ll}0.939 & 1.065\end{array}\right]$} & {$\left[\begin{array}{ll}0.866 & 1.137\end{array}\right]$} & {$\left[\begin{array}{ll}0.890 & 1.134\end{array}\right]$} & {$\left[\begin{array}{ll}0.908 & 1.108\end{array}\right]$} & {$\left[\begin{array}{ll}0.818 & 1.196\end{array}\right]$} \\
\hline \multirow[t]{2}{*}{$\sigma_{E G A R C H-S P}$} & $3.540(0.326)$ & $4.618(0.291)$ & $3.285(0.622)$ & $4.335(0.406)$ & $5.338(0.367)$ & $4.121(0.915)$ & $5.972(0.754)$ & $6.841(0.689)$ & $4.893(1.402)$ \\
\hline & {$[0.8531 .156]$} & {$\left[\begin{array}{ll}0.901 & 1.105\end{array}\right]$} & {$\left[\begin{array}{ll}0.905 & 1.092\end{array}\right]$} & {$\left[\begin{array}{ll}0.849 & 1.166\end{array}\right]$} & {$[0.8881 .119]$} & {$[0.877$ 1.129] } & {$\left[\begin{array}{ll}0.805 & 1.226\end{array}\right]$} & {$\left[\begin{array}{ll}0.848 & 1.186\end{array}\right]$} & {$\left[\begin{array}{ll}0.823 & 1.184\end{array}\right]$} \\
\hline \multirow[t]{2}{*}{$\sigma_{G J R-S P}$} & $3.595(0.316)$ & $4.650(0.284)$ & $3.344(0.604)$ & $4.373(0.394)$ & $5.356(0.363)$ & $4.163(0.911)$ & $5.977(0.739)$ & $6.827(0.675)$ & $4.920(1.373)$ \\
\hline & {$[0.8601 .151]$} & {$\left[\begin{array}{ll}0.903 & 1.106\end{array}\right]$} & {$\left[\begin{array}{ll}0.908 & 1.096\end{array}\right]$} & {$[0.8551 .155]$} & {$[0.8911 .119]$} & {$\left[\begin{array}{ll}0.874 & 1.131]\end{array}\right.$} & {$\left[\begin{array}{ll}0.813 & 1.228\end{array}\right]$} & {$\left[\begin{array}{ll}0.849 & 1.184\end{array}\right]$} & {$[0.8211 .192]$} \\
\hline
\end{tabular}




\section{Table 3: Risk estimates for FTSE100.}

\begin{tabular}{|c|c|c|c|c|c|c|c|c|c|}
\hline Model & $\operatorname{VaR}(0.99)$ & $\mathrm{ES}(0.99)$ & $\operatorname{SRM}(50)$ & $\operatorname{VaR}(0.995)$ & $\mathrm{ES}(0.995)$ & SRM(100) & $\operatorname{VaR}(0.999)$ & $\mathrm{ES}(0.999)$ & $\operatorname{SRM}(200)$ \\
\hline \multicolumn{10}{|c|}{ Panel A: Long position } \\
\hline Empirical & 3.846 & 5.731 & 4.171 & 5.074 & 7.128 & 5.438 & 8.604 & 9.384 & 7.012 \\
\hline \multirow[t]{2}{*}{$\sigma_{\text {constant }}$} & $3.308(0.141)$ & $3.787(0.126)$ & $3.194(0.190)$ & $3.661(0.180)$ & $4.107(0.162)$ & $3.566(0.292)$ & $4.389(0.334)$ & $4.773(0.300)$ & $3.909(0.443)$ \\
\hline & {$\left[\begin{array}{ll}0.931 & 1.073\end{array}\right]$} & {$\left[\begin{array}{ll}0.947 & 1.057\end{array}\right]$} & {$\left[\begin{array}{ll}0.903 & 1.102\end{array}\right]$} & {$\left[\begin{array}{ll}0.920 & 1.085\end{array}\right]$} & {$\left[\begin{array}{ll}0.938 & 1.068\end{array}\right]$} & {$\left[\begin{array}{ll}0.869 & 1.138\end{array}\right]$} & {$[0.8851 .136]$} & {$\left[\begin{array}{ll}0.907 & 1.116\end{array}\right]$} & {$\left[\begin{array}{ll}0.816 & 1.198\end{array}\right]$} \\
\hline \multirow[t]{2}{*}{$\sigma_{G A R C H-S P}$} & $3.525(0.166)$ & $4.010(0.151)$ & $3.389(0.244)$ & $3.949(0.217)$ & $4.484(0.200)$ & $3.835(0.377)$ & $4.823(0.403)$ & $5.284(0.368)$ & $4.247(0.579)$ \\
\hline & {$\left[\begin{array}{ll}0.923 & 1.080\end{array}\right]$} & {$\left[\begin{array}{ll}0.942 & 1.063\end{array}\right]$} & {$\left[\begin{array}{ll}0.906 & 1.098\end{array}\right]$} & {$\left[\begin{array}{ll}0.914 & 1.098\end{array}\right]$} & {$\left[\begin{array}{ll}0.929 & 1.077\end{array}\right]$} & {$\left[\begin{array}{ll}0.870 & 1.137\end{array}\right]$} & {$[0.876$ 1.153] } & {$[0.894 \quad 1.126]$} & {$\left[\begin{array}{ll}0.819 & 1.193\end{array}\right]$} \\
\hline \multirow[t]{2}{*}{$\sigma_{E G A R C H-S P}$} & $3.574(0.290)$ & $4.546(0.256)$ & $3.344(0.543)$ & $4.291(0.362)$ & $5.196(0.321)$ & $4.098(0.801)$ & $5.768(0.685)$ & $6.550(0.611)$ & $4.794(1.232)$ \\
\hline & {$[0.867 \quad 1.142]$} & {$\left[\begin{array}{ll}0.910 & 1.097\end{array}\right]$} & {$\left[\begin{array}{ll}0.908 & 1.094\end{array}\right]$} & {$[0.864$ 1.147] } & {$[0.901 \quad 1.108]$} & {$[0.874$ 1.130] } & {$\left[\begin{array}{ll}0.822 & 1.213\end{array}\right]$} & {$\left[\begin{array}{ll}0.855 & 1.169\end{array}\right]$} & {$\left[\begin{array}{ll}0.818 & 1.189\end{array}\right]$} \\
\hline \multirow[t]{2}{*}{$\sigma_{G J R-S P}$} & $3.722(0.231)$ & $4.518(0.211)$ & $3.534(0.404)$ & $4.309(0.297)$ & $5.050(0.273)$ & $4.151(0.610)$ & $5.518(0.547)$ & $6.158(0.496)$ & $4.721(0.907)$ \\
\hline & {$[0.899 \quad 1.107]$} & {$\left[\begin{array}{ll}0.924 & 1.079\end{array}\right]$} & {$[0.9051 .096]$} & {$\left[\begin{array}{ll}0.891 & 1.121\end{array}\right]$} & {$\left[\begin{array}{ll}0.916 & 1.093\end{array}\right]$} & {$[0.874$ 1.137] } & {$[0.847 \quad 1.176]$} & {$[0.877 \quad 1.146]$} & {$\left[\begin{array}{ll}0.825 & 1.187\end{array}\right]$} \\
\hline \multicolumn{10}{|c|}{ Panel B: Short position } \\
\hline Empirical & 4.593 & 5.882 & 4.355 & 5.415 & 6.738 & 5.532 & 8.338 & 9.265 & 6.920 \\
\hline \multirow[t]{2}{*}{$\sigma_{\text {constant }}$} & $3.278(0.141)$ & $3.757(0.126)$ & $3.164(0.187)$ & $3.631(0.179)$ & $4.077(0.163)$ & $3.536(0.290)$ & $4.359(0.337)$ & $4.744(0.303)$ & $3.879(0.450)$ \\
\hline & {$\left[\begin{array}{ll}0.931 & 1.071]\end{array}\right]$} & {$\left[\begin{array}{ll}0.945 & 1.056\end{array}\right]$} & {$\left[\begin{array}{ll}0.904 & 1.099\end{array}\right]$} & {$\left[\begin{array}{ll}0.921 & 1.085\end{array}\right]$} & {$\left[\begin{array}{ll}0.936 & 1.067\end{array}\right]$} & {$[0.8691 .133]$} & {$\left[\begin{array}{ll}0.884 & 1.139\end{array}\right]$} & {$\left[\begin{array}{ll}0.903 & 1.115\end{array}\right]$} & {$\left[\begin{array}{ll}0.817 & 1.198\end{array}\right]$} \\
\hline \multirow[t]{2}{*}{$\sigma_{G A R C H-S P}$} & $3.124(0.126)$ & $3.550(0.113)$ & $3.023(0.155)$ & $3.438(0.160)$ & $3.835(0.1465)$ & $3.353(0.244)$ & $4.086(0.303)$ & $4.427(0.265)$ & $3.659(0.371)$ \\
\hline & {$[0.9351 .070]$} & {$[0.948$ 1.054] } & {$\left[\begin{array}{ll}0.906 & 1.101\end{array}\right]$} & {$[0.926$ 1.082] } & {$\left[\begin{array}{ll}0.939 & 1.067\end{array}\right]$} & {$\left[\begin{array}{ll}0.867 & 1.137\end{array}\right]$} & {$\left[\begin{array}{ll}0.889 & 1.134\end{array}\right]$} & {$\left[\begin{array}{ll}0.909 & 1.108\end{array}\right]$} & {$\left[\begin{array}{ll}0.815 & 1.191\end{array}\right]$} \\
\hline \multirow[t]{2}{*}{$\sigma_{E G A R C H-S P}$} & $3.545(0.285)$ & $4.516(0.255)$ & $3.314(0.536)$ & $4.261(0.367)$ & $5.166(0.328)$ & $4.068(0.809)$ & $5.738(0.679)$ & $6.520(0.610)$ & $4.765(1.215)$ \\
\hline & {$[0.8661 .138]$} & {$\left[\begin{array}{ll}0.908 & 1.095\end{array}\right]$} & {$\left[\begin{array}{ll}0.909 & 1.092\end{array}\right]$} & {$\left[\begin{array}{ll}0.862 & 1.150\end{array}\right]$} & {$[0.899 \quad 1.111]$} & {$\left[\begin{array}{ll}0.872 & 1.131\end{array}\right]$} & {$\left[\begin{array}{ll}0.819 & 1.214\end{array}\right]$} & {$[0.857$ 1.169] } & {$\left[\begin{array}{ll}0.821 & 1.189\end{array}\right]$} \\
\hline \multirow[t]{2}{*}{$\sigma_{G J R-S P}$} & $3.693(0.231)$ & $4.488(0.212)$ & $3.504(0.399)$ & $4.279(0.296)$ & $5.020(0.266)$ & $4.121(0.606)$ & $5.489(0.548)$ & $6.129(0.508)$ & $4.692(0.932)$ \\
\hline & {$[0.8961 .107]$} & {$[0.9251 .083]$} & {$[0.908$ 1.094] } & {$[0.888 \quad 1.120]$} & {$[0.915 \quad 1.091]$} & {$[0.8721 .133]$} & {$\left[\begin{array}{ll}0.851 & 1.181]\end{array}\right.$} & {$[0.8741 .148]$} & {$\left[\begin{array}{ll}0.819 & 1.194\end{array}\right]$} \\
\hline
\end{tabular}

Note: Performance of risk measures for both long position (Panel A) and short position (Panel B) of FTSE100. SE's are reported besides each estimate. CI's are reported below. 
Table 4: Risk estimates for DAX

\begin{tabular}{|c|c|c|c|c|c|c|c|c|c|}
\hline Model & $\operatorname{VaR}(0.99)$ & $\mathrm{ES}(0.99)$ & $\operatorname{SRM}(50)$ & $\operatorname{VaR}(0.995)$ & $\mathrm{ES}(0.995)$ & $\operatorname{SRM}(100)$ & $\operatorname{VaR}(0.999)$ & $\mathrm{ES}(0.999)$ & $\operatorname{SRM}(200)$ \\
\hline \multicolumn{10}{|c|}{ Panel A: Long position } \\
\hline Empirical & 4.639 & 6.410 & 4.787 & 5.195 & 7.867 & 6.161 & 10.700 & 10.798 & 7.919 \\
\hline \multirow{2}{*}{$\sigma_{\text {constant }}$} & $3.832(0.165)$ & $4.385(0.143)$ & $3.701(0.213)$ & $4.240(0.206)$ & $4.755(0.190)$ & $4.1299(0.336)$ & $5.082(0.391)$ & $5.526(0.343)$ & $4.527(0.508)$ \\
\hline & {$[0.9301 .072]$} & {$[0.9481 .056]$} & [ $0.907 \quad 1.099]$ & {$\left[\begin{array}{ll}0.922 & 1.084\end{array}\right]$} & {$\left[\begin{array}{ll}0.935 & 1.069\end{array}\right]$} & {$\left[\begin{array}{ll}0.867 & 1.139\end{array}\right]$} & {$\left[\begin{array}{ll}0.886 & 1.140\end{array}\right]$} & {$[0.904$ 1.112] } & {$\left[\begin{array}{ll}0.822 & 1.192\end{array}\right]$} \\
\hline \multirow{2}{*}{$\sigma_{G A R C H-S P}$} & $3.955(0.183)$ & $4.580(0.162)$ & $3.807(0.260)$ & $4.416(0.234)$ & $4.998(0.214)$ & $4.292(0.408)$ & $5.366(0.435)$ & $5.867(0.391)$ & $4.740(0.623)$ \\
\hline & {$\left[\begin{array}{ll}0.925 & 1.080\end{array}\right]$} & {$\left[\begin{array}{ll}0.943 & 1.060\end{array}\right]$} & {$\left[\begin{array}{ll}0.905 & 1.096\end{array}\right]$} & {$\left[\begin{array}{ll}0.916 & 1.092\end{array}\right]$} & {$\left[\begin{array}{ll}0.932 & 1.073\end{array}\right]$} & {$\left[\begin{array}{ll}0.866 & 1.139\end{array}\right]$} & {$\left[\begin{array}{ll}0.878 & 1.143\end{array}\right]$} & {$[0.898$ 1.119] } & {$\left[\begin{array}{ll}0.815 & 1.199\end{array}\right]$} \\
\hline \multirow[t]{2}{*}{$\sigma_{E G A R C H-S P}$} & $4.068(0.237)$ & $4.877(0.212)$ & $3.876(0.392)$ & $4.665(0.303)$ & $5.418(0.274)$ & $4.504(0.585)$ & $5.894(0.566)$ & $6.545(0.509)$ & $5.084(0.904)$ \\
\hline & {$\left[\begin{array}{ll}0.906 & 1.101\end{array}\right]$} & {$\left[\begin{array}{ll}0.930 & 1.073\end{array}\right]$} & {$\left[\begin{array}{ll}0.907 & 1.094\end{array}\right]$} & {$\left[\begin{array}{ll}0.897 & 1.113\end{array}\right]$} & {$\left[\begin{array}{ll}0.919 & 1.089\end{array}\right]$} & {$\left[\begin{array}{ll}0.876 & 1.129\end{array}\right]$} & {$[0.8541 .172]$} & {$\left[\begin{array}{ll}0.883 & 1.141\end{array}\right]$} & {$\left[\begin{array}{ll}0.820 & 1.186\end{array}\right]$} \\
\hline \multirow[t]{2}{*}{$\sigma_{G J R-S P}$} & $4.070(0.227)$ & $4.852(0.205)$ & $3.885(0.377)$ & $4.647(0.293)$ & $5.375(0.265)$ & $4.491(0.568)$ & $5.836(0.552)$ & $6.465(0.497)$ & $5.052(0.860)$ \\
\hline & {$[0.9091 .096]$} & {$[0.934 \quad 1.074]$} & {$[0.906 \quad 1.099]$} & {$[0.901 \quad 1.108]$} & {$\left[\begin{array}{ll}0.921 & 1.087\end{array}\right]$} & {$[0.8691 .133]$} & {$[0.856 \quad 1.171]$} & {$\left[\begin{array}{ll}0.883 & 1.140\end{array}\right]$} & {$\left[\begin{array}{ll}0.823 & 1.184\end{array}\right]$} \\
\hline \multicolumn{10}{|c|}{ Panel B: Short position } \\
\hline Empirical & 5.192 & 5.947 & 4.728 & 5.808 & 6.645 & 5.714 & 7.279 & 7.336 & 6.709 \\
\hline \multirow[t]{2}{*}{$\sigma_{\text {constant }}$} & $3.780(0.162)$ & $4.333(0.145)$ & $3.648(0.222)$ & $4.188(0.211)$ & $4.703(0.191)$ & $4.078(0.343)$ & $5.029(0.383)$ & $5.473(0.344)$ & $4.474(0.520)$ \\
\hline & {$\left[\begin{array}{ll}0.930 & 1.073\end{array}\right]$} & {$\left[\begin{array}{ll}0.945 & 1.056\end{array}\right]$} & {$[0.901 \quad 1.102]$} & {$\left[\begin{array}{ll}0.919 & 1.086\end{array}\right]$} & {$\left[\begin{array}{ll}0.936 & 1.070\end{array}\right]$} & {$\left[\begin{array}{ll}0.864 & 1.139\end{array}\right]$} & {$\left[\begin{array}{ll}0.885 & 1.135\end{array}\right]$} & {$\left[\begin{array}{ll}0.905 & 1.117]\end{array}\right.$} & {$\left[\begin{array}{ll}0.815 & 1.197\end{array}\right]$} \\
\hline \multirow[t]{2}{*}{$\sigma_{G A R C H-S P}$} & $3.645(0.143)$ & $4.139(0.129)$ & $3.527(0.183)$ & $4.009(0.188)$ & $4.470(0.170)$ & $3.911(0.278)$ & $4.761(0.347)$ & $5.158(0.315)$ & $4.266(0.433)$ \\
\hline & {$\left[\begin{array}{ll}0.937 & 1.068\end{array}\right]$} & {$\left[\begin{array}{ll}0.949 & 1.054\end{array}\right]$} & {$\left[\begin{array}{ll}0.903 & 1.101\end{array}\right]$} & {$\left[\begin{array}{ll}0.926 & 1.081\end{array}\right]$} & {$\left[\begin{array}{ll}0.940 & 1.066\end{array}\right]$} & {$[0.8691 .132]$} & {$\left[\begin{array}{ll}0.890 & 1.130\end{array}\right]$} & {$\left[\begin{array}{ll}0.906 & 1.110\end{array}\right]$} & {$\left[\begin{array}{ll}0.818 & 1.194\end{array}\right]$} \\
\hline \multirow[t]{2}{*}{$\sigma_{E G A R C H-S P}$} & $4.016(0.236)$ & $4.825(0.213)$ & $3.824(0.396)$ & $4.612(0.302)$ & $5.366(0.273)$ & $4.452(0.599)$ & $5.842(0.562)$ & $6.492(0.513)$ & $5.031(0.905)$ \\
\hline & {$\left[\begin{array}{ll}0.904 & 1.099\end{array}\right]$} & {$\left[\begin{array}{ll}0.931 & 1.076\end{array}\right]$} & {$\left[\begin{array}{ll}0.906 & 1.094\end{array}\right]$} & {$\left[\begin{array}{ll}0.895 & 1.114\end{array}\right]$} & {$\left[\begin{array}{ll}0.917 & 1.089\end{array}\right]$} & {$\left[\begin{array}{ll}0.873 & 1.135\end{array}\right]$} & {$\left[\begin{array}{ll}0.853 & 1.169\end{array}\right]$} & {$[0.8801 .142]$} & {$\left[\begin{array}{ll}0.821 & 1.187\end{array}\right]$} \\
\hline \multirow[t]{2}{*}{$\sigma_{G J R-S P}$} & $4.018(0.227)$ & $4.800(0.206)$ & $3.832(0.377)$ & $4.594(0.291)$ & $5.323(0.266)$ & $4.439(0.574)$ & $5.784(0.546)$ & $6.412(0.489)$ & $4.999(0.856)$ \\
\hline & {$\left[\begin{array}{ll}0.909 & 1.096\end{array}\right]$} & {$\left[\begin{array}{ll}0.930 & 1.074\end{array}\right]$} & {$\left[\begin{array}{ll}0.903 & 1.097\end{array}\right]$} & {$[0.899 \quad 1.110]$} & {$\left[\begin{array}{ll}0.922 & 1.087\end{array}\right]$} & {$[0.874$ 1.139] } & {$\left[\begin{array}{ll}0.854 & 1.168]\end{array}\right.$} & {$\left[\begin{array}{ll}0.883 & 1.138\end{array}\right]$} & {$\left[\begin{array}{ll}0.820 & 1.190\end{array}\right]$} \\
\hline
\end{tabular}

Note: Performance of risk measures for both long position (Panel A) and short position (Panel B) of DAX. SE's are reported besides each estimate. CI's are reported below. 